\title{
Experimental investigation of the neutral sheet profile during magnetic reconnection
}

\author{
Masaaki Yamada, Hantao Ji, Scott Hsu, Troy Carter, Russell Kulsrud, and \\ Fedor Trintchouk \\ Princeton Plasma Physics Laboratory, P. O. Box 451, Princeton, NJ 08543
}

\begin{abstract}
During magnetic reconnection, a "neutral sheet" current is induced, heating the plasma. The resultant plasma thermal pressure forms a stationary equilibrium with the opposing magnetic fields. The reconnection layer profile holds significant clues about the physical mechanisms which control reconnection. On the Magnetic Reconnection Experiment [M. Yamada et al., Phys. Plasmas 4, 1936 (1997)], a quasi steady-state and axisymmetric neutral sheet profile has been measured precisely using a magnetic probe array with spatial resolution equal to one quarter of the ion gyro-radius. It was found that the reconnecting field profile fits well with a Harris-type profile [E. G. Harris, Il Nuovo Cimento 23, $115(1962)], B(x) \sim \tanh (x / \delta)$. This agreement is remarkable since the Harris theory does not take into account reconnection and associated electric fields and dissipation. An explanation for this agreement is presented. The sheet thickness $\delta$ is found to be approximately 0.4 times the ion skin depth, which agrees with a generalized Harris theory incorporating non-isothermal electron and ion temperatures and finite electric field. The detailed study of additional local features of the reconnection region is also presented.
\end{abstract}

PACS numbers: 52.30.Jb, 96.60.Rd, 94.30.Lr

Typeset using REVTEX 


\section{INTRODUCTION}

Magnetic reconnection, ${ }^{1}$ the topological rearrangement of magnetic field lines, is a basic plasma phenomena of wide-ranging importance. It plays a major role in the evolution of solar flares ${ }^{2}$ and in the interaction of the solar wind with the earth's magnetosphere, ${ }^{3}$ and it often plays a dominant role in determining the confinement characteristics of hightemperature fusion plasmas. ${ }^{4}$ Recent detailed data from laboratory plasma experiments, satellite observations, theoretical analysis, and computer simulations have advanced the understanding of magnetic reconnection both in space and laboratory plasmas.

The reconnection layer, often called the neutral sheet, is a focal point of reconnection research since magnetohydrodynamics (MHD) breaks down locally in the layer, while globally, the plasma has large Lundquist number and is approximated well by ideal MHD. Precise measurements of the neutral sheet profile can provide important clues to help understand the physical mechanisms of reconnection. Thanks to significant progress in data acquisition technology in the past two decades, the detailed magnetic field structure of the neutral sheet has been measured in laboratory plasmas. Extensive data have been accumulated in highly conductive MHD plasmas with large Lundquist numbers $S=10-1000$, where $S$ is the ratio of the magnetic diffusion time to the Alfvén transit time.

MRX $^{5}$ (Magnetic Reconnection Experiment) was built in 1995 at the Princeton Plasma Physics Laboratory for the comprehensive study of magnetic reconnection in a controlled environment. It has generated much fundamental data on magnetic reconnection in MHD plasmas. Our experiment has already addressed important issues related to the reconnection layer. Based on detailed magnetic field measurements by high-resolution magnetic probes, evolution of poloidal flux contours were deduced, demonstrating important two-dimensional (2-D) features of magnetic reconnection. ${ }^{6}$ The reconnection rate was also seen to agree with a generalized Sweet-Parker model. ${ }^{7,8}$

Generally, analysis of magnetic reconnection is divided into local and global issues. In local treatments, the reconnection rate is determined by local plasma parameters in the 
reconnection region. In these analyses, boundaries are not specified and boundary conditions are often replaced by an initial condition for plasma flow velocity. In global treatments, on the other hand, the reconnection rate is considered to be influenced or determined by the three-dimensional (3-D) topology of the plasma configuration. Boundaries are well defined and the conservation of total flux or helicity is often discussed. Three dimensional MHD modes are often involved, and particle acceleration in all three dimensions are possible. The present paper addresses the most fundamental local reconnection physics issues by studying the features of the reconnection layer in MRX.

As magnetic field lines break and reconnect around the neutral layer, a current sheet is generated, heating the plasma. The plasma thermal pressure then forms an equilibrium with the magnetic pressure of the reconnecting magnetic field. While MHD can be used to treat the plasma globally, more general theories are necessary for a proper treatment of the neutral layer, where MHD breaks down. An important question is what determines the profile of the reconnection layer. Harris $^{9}$ found analytical one-dimensional (1-D) solutions for the magnetic field, plasma pressure, current density, and sheet thickness of a collisionless current sheet by solving the Vlasov-Maxwell equations with three important assumptions: (1) no electric field; (2) electrons and ions drifting in opposite directions at the diamagnetic speed; and (3) equal and spatially uniform electron and ion temperatures. The Harris solution is an elegant description of a neutral sheet and therefore is referenced very often in reconnection research. Numerical simulations have yielded ${ }^{10-13}$ favorable comparisons with the Harris sheet, including the sheet thickness being equal to the ion skin-depth. ${ }^{13}$ However, to our knowledge, the Harris sheet has not been investigated experimentally. The precise determination of magnetic field profiles in MRX has enabled a detailed study of the current sheet thickness as a function of relevant parameters, which is the subject of this paper.

The paper is organized as follows. Sec. II presents a generalized theory of the Harris collisionless neutral sheet. Sec. III describes the experimental apparatus of MRX. Sec. IV presents detailed experimental results on the neutral sheet profile during reconnection. Sec. V concludes the paper with a summary and discussion. 


\section{THEORY OF A GENERALIZED HARRIS SHEET}

A steady-state, collisionless current sheet can be analyzed using the steady-state VlasovMaxwell system of equations

$$
\begin{aligned}
& \boldsymbol{v} \cdot \frac{\partial f}{\partial \boldsymbol{r}}+\frac{q}{m}(\boldsymbol{E}+\boldsymbol{v} \times \boldsymbol{B}) \cdot \frac{\partial f}{\partial \boldsymbol{v}}=0 \\
& \boldsymbol{\nabla} \times \boldsymbol{E}=0 \\
& \boldsymbol{\nabla} \cdot \boldsymbol{E}=\frac{e}{\epsilon_{0}}\left(\int f_{i} d \boldsymbol{v}-\int f_{e} d \boldsymbol{v}\right) \\
& \boldsymbol{\nabla} \times \boldsymbol{B}=e \mu_{0}\left(\int \boldsymbol{v} f_{i} d \boldsymbol{v}-\int \boldsymbol{v} f_{e} d \boldsymbol{v}\right) \\
& \boldsymbol{\nabla} \cdot \boldsymbol{B}=0,
\end{aligned}
$$

where $\boldsymbol{E}$ is the electric field, $\boldsymbol{B}$ the magnetic field, $\boldsymbol{v}$ the particle velocity, and $f_{i}\left(f_{e}\right)$ the ion (electron) distribution function. In general, the equations are nonlinear and can only be solved numerically. However, E. G. Harris $^{9}$ derived a 1-D steady-state analytical solution with certain assumptions. Below, we re-derive the Harris solution, relaxing a few of the original assumptions.

All solutions of Eq. (1) must be a function of the constants of the motion, i.e. the total energy $W \equiv m v^{2} / 2 \pm e \phi$ and the canonical momentum in the $y$ and $z$ directions,

$p_{y} \equiv m v_{y} \pm e A_{y}$ and $p_{z} \equiv m v_{z}$, respectively. Here, $\phi$ is the electrostatic potential and the vector potential $\boldsymbol{A}$ is assumed to have only a $y$-component $A_{y}$. Consider the distribution function

$$
f=n_{0}\left(\frac{m}{2 \pi T}\right)^{3 / 2} \exp \left\{-\frac{m\left[v_{x}^{2}+\left(v_{y}-V\right)^{2}+v_{z}^{2}\right]}{2 T} \pm \frac{e\left(V A_{y}-\phi\right)}{T}\right\}
$$

where $T=T_{e}\left(T_{i}\right)$ is the electron (ion) temperature and $V=V_{e}\left(V_{i}\right)$ is the electron (ion) drift speed in the $y$ direction. Since the argument of the exponential can be written as $\left(-W+p_{y} V-m V^{2} / 2\right) / T$, then $f$ is a function of the constants of the motion and therefore a solution of Eq. (1). Harris realized that this shifted Maxwellian is the most natural solution. Here, we have relaxed Harris' assumptions $T_{e}=T_{i}$ and $V_{i}=-V_{e}$. 
In this 1-D model, all variables are assumed to vary only in $x$ except $T_{i}, T_{e}, V_{i}$, and $V_{e}$ which are all assumed to be constant. The $y$ direction is the direction of the current. The system of equations can be simplified significantly by assuming that $\boldsymbol{E}$ has only an $x$-component $E_{x}=-\partial \phi / \partial x$. And since $A_{y}$ is assumed to be the only non-zero component of $\boldsymbol{A}, \boldsymbol{B}$ has only a $z$-component $B_{z}=\partial A_{y} / \partial x$. It should be noted that the $E_{y}=0$ and collisionless assumptions mean that there is no dissipation and hence no reconnection in this model.

Substitution of Eq. (6) into Eqs. (3) and (4) yields two coupled nonlinear differential equations for $\phi$ and $A_{y}$,

$$
\begin{aligned}
\frac{\partial^{2} \phi}{\partial x^{2}} & =-\frac{e n_{0}}{\epsilon_{0}}\left\{\exp \left[\frac{e\left(V_{i} A_{y}-\phi\right)}{T_{i}}\right]-\exp \left[\frac{-e\left(V_{e} A_{y}-\phi\right)}{T_{e}}\right]\right\} \\
\frac{\partial^{2} A_{y}}{\partial x^{2}} & =-e n_{0} \mu_{0}\left\{V_{i} \exp \left[\frac{e\left(V_{i} A_{y}-\phi\right)}{T_{i}}\right]-V_{e} \exp \left[\frac{-e\left(V_{e} A_{y}-\phi\right)}{T_{e}}\right]\right\} .
\end{aligned}
$$

By using normalized variables $\hat{\phi} \equiv e \phi / T_{e}$ and $\hat{x} \equiv x /\left(c / \omega_{\mathrm{pi}}\right)\left(\right.$ where $\omega_{\mathrm{pi}} \equiv n_{0} e^{2} / \epsilon_{0} m_{i}$ is the ion plasma frequency), Eq. (7) is cast into dimensionless form,

$$
\frac{\partial^{2} \hat{\phi}}{\partial \hat{x}^{2}}=-\left(\frac{c / \omega_{\mathrm{pi}}}{\lambda_{D}}\right)^{2}\left\{\exp \left[\frac{e\left(V_{i} A_{y}-\phi\right)}{T_{i}}\right]-\exp \left[\frac{-e\left(V_{e} A_{y}-\phi\right)}{T_{e}}\right]\right\}
$$

where the left-hand side (LHS) is of order unity but $\left(c / \omega_{\mathrm{pi}} / \lambda_{D}\right)^{2}$ is of order $10^{6}\left(\lambda_{D}\right.$ is the Debye length). Therefore, quasineutrality $\{\cdots\} \simeq 0$ must be satisfied, leading to an ambipolar potential

$$
\phi=\frac{T_{e} V_{i}+T_{i} V_{e}}{T_{e}+T_{i}} A_{y}
$$

Interestingly, $\phi$ is proportional to $A_{y}$, and substituting Eq. (10) into Eq. (8) gives a nonlinear equation in only $A_{y}$,

$$
\frac{\partial^{2} A_{y}}{\partial x^{2}}=-e n_{0} \mu_{0}\left(V_{i}-V_{e}\right) \exp \left[\frac{e\left(V_{i}-V_{e}\right)}{T_{e}+T_{i}} A_{y}\right] .
$$

With appropriate boundary conditions, this equation can be solved analytically, yielding modified Harris solutions, 


$$
\begin{aligned}
A_{y} & =-\delta B_{0} \log \cosh \left(\frac{x}{\delta}\right) \\
B_{z} & =-B_{0} \tanh \left(\frac{x}{\delta}\right) \\
j_{y} & =\frac{B_{0}}{\mu_{0} \delta} \operatorname{sech}^{2}\left(\frac{x}{\delta}\right) \\
E_{x} & =\frac{T_{e} V_{i}+T_{i} V_{e}}{T_{e}+T_{i}} B_{0} \tanh \left(\frac{x}{\delta}\right) \\
p & =n_{0}\left(T_{e}+T_{i}\right) \operatorname{sech}^{2}\left(\frac{x}{\delta}\right),
\end{aligned}
$$

where $B_{0}^{2} /\left(2 \mu_{0}\right)=n_{0}\left(T_{e}+T_{i}\right)$. The current sheet thickness $\delta$ is given by

$$
\delta=\frac{c}{\omega_{\mathrm{pi}}} \frac{\sqrt{2\left(T_{e}+T_{i}\right) / m_{i}}}{V_{i}-V_{e}}=\frac{c}{\omega_{\mathrm{pi}}} \frac{\sqrt{2} V_{\mathrm{s}}}{V_{\mathrm{drift}}},
$$

where $V_{\mathrm{s}} \equiv \sqrt{\left(T_{e}+T_{i}\right) / m_{i}}$ and $V_{\mathrm{drift}} \equiv V_{i}-V_{e}$ is the relative drift between ions and electrons. It should be noted that the above solution is more general than the original Harris solution, which is limited to $E_{x}=\phi=0$. The original Harris solution can be recovered by setting $T_{e}=T_{i}=T$ and $V_{i}=-V_{e}=V$ in Eq. (17) to yield $\delta=\left(c / \omega_{\mathrm{pi}}\right)\left(\sqrt{T / m_{i}} / V\right)$.

One could find solutions other than Eq. (6) by choosing other functions of the constants of the motion, which would lead to magnetic field profiles different from Eq. (13). However, because the Harris solution for $f$ is a shifted Maxwellian, the ion-ion and electron-electron collision integrals vanish. Thus, it is the most natural solution of the Vlasov-Maxwell equations for the reconnection geometry. The electron-ion collision integral does not vanish but gives rise to the resistivity term $\eta j_{y}$. This term is balanced by an induced electric field $E_{y}$, which produces an additional flow in $x$ and brings magnetic field lines into the layer to be reconnected. The ion-ion and electron-electron collision terms in the Fokker-Planck equation force the distribution function to be a local Maxwellian as well as a function of only the constants of the motion. The electron-ion collision term, once it is balanced by the induced electric field, produces only a small correction to the distribution function in Eq. (6). And the constant temperature assumption is justified at least for the ions by the rapid thermal conduction in the $x$ direction when the ion gyro-radius is comparable to $\delta$. In the case of the MRX experiment, $T_{e}$ and $T_{i}$ were measured to be almost constant in the neutral sheet. The drift velocity $V$, not specified in the Harris solution, is determined by 
additional reconnection physics, i.e. the outflow of plasma from the layer must be balanced by incoming flow through the electron-ion collision term. If the resistivity is anomalous, the remarks above still apply with the resistivity replaced by the anomalous resistivity.

The Harris model has been generalized further by S. M. Mahajan ${ }^{14}$ to include timedependence, cylindrical geometry, and various density and velocity profiles. Generally, the solutions must be determined numerically. However, when ions drift in the toroidal direction ( $y$ direction in Cartesian coordinates) with constant angular velocity $\omega=V_{i \theta} / r$, an analytical solution is found, ${ }^{15}$ with

$$
B_{z}=B_{0} \tanh \left(\frac{r^{2}-r_{0}^{2}}{r_{0} \delta}\right)
$$

where $r_{0}$ is the radial position of the center of the current sheet.

The "Harris sheet" solution is a simple analytical equilibrium solution for a plasma confined between oppositely directed magnetic fields. The convenient analytical expressions for the $B_{z}, j_{y}$, and $p$ profiles and the sheet thickness $\delta$ lend themselves to direct comparisons with both computer simulations and experiments. However, it is important to keep in mind the limitations and assumptions of the Harris model, including the fact that it is 1-D and does not include the effects of reconnection and associated electric fields, and to find out how reconnection and 2-D effects modify the Harris equilibrium.

\section{EXPERIMENTAL APPARATUS}

Experiments for this research are performed in the MRX device. ${ }^{5}$ The attainable magnetic Lundquist number $S$ is 300-1000. Figure 1(a) shows the present experimental setup in MRX. Two flux cores with $37.5 \mathrm{~cm}$ major radii and $9.4 \mathrm{~cm}$ minor radii are installed in the vacuum vessel. Inside each core, there is a 4-turn, PF coil that carries toroidal current and a helical 36-turn toroidal solenoid (TF coil) that also acts as a one-turn toroidal current coil. By properly programming currents in the PF and TF coils, plasma current and toroidal field can be prescribed, independent of the sense of helicity in the toroidal solenoid. The coils 
are powered by two sets of capacitor banks, each of them containing eight $60 \mu \mathrm{F}, 20 \mathrm{kV}$ capacitors and 50-100 kJ of stored energy.

The present experiments are conducted in the double annular plasma setup, in which two toroidal plasmas with annular cross sections are formed independently around the flux cores. Magnetic reconnection is then driven in the quadrupole field. By pulsing currents in the TF coils after a quadrupole poloidal magnetic field has been established by the PF coil currents, plasmas are created around each flux core due to inductive poloidal electric fields. At the same time, a common annular plasma forms outside the two inner plasmas surrounding each flux core. Thus, the magnetic field domain can be divided into a public domain and two private domains. Once the annular plasmas are created, the PF coil current can be increased or decreased. In the case of increasing PF coil current, the poloidal flux in each plasma is "pushed" toward the X-point (push mode). In the case of decreasing PF coil current, the poloidal flux in the public region is "pulled" back toward the x-point (pull mode), as illustrated in Fig. 1(b).

In the present MRX experiments, "pull" mode has been utilized without an magnetic axial field [the third vector component, in the toroidal direction $\theta$ in Fig. 1(a)]. In this so-called "null-helicity" reconnection, the pitch of the field lines changes sharply at the reconnection point. Figure 1(c) shows the time evolution of measured poloidal flux contours during null-helicity reconnection. It should be noted that the pull mode geometry shares some common geometrical features with the plasma sheet at the magnetotail, in which time variation of the ring current and cross-tail currents induces magnetic reconnection, as well as with helmet streamer structures in the solar corona.

To document the internal magnetic structure of the reconnection in a single shot, twodimensional magnetic probe arrays are placed in an $R-Z$ plane, as shown in Fig. 1(a). Density and electron temperature $T_{e}$ are measured using a triple Langmuir probe. Ion temperature $T_{i}$ is estimated using Doppler spectroscopy of the $\mathrm{H}_{\beta}$ line, based on fast chargeexchange times on the order of $1 \mu \mathrm{s}$, and ion flows are measured using a Mach probe. Plasma parameters are as follows: $B \sim 0.5-1.0 \mathrm{kG}, n_{e} \sim 0.1-2.0 \times 10^{14} \mathrm{~cm}^{-3}, T_{e} \sim 5-20 \mathrm{eV}$, and 
$T_{i} \sim 10-30 \mathrm{eV}$.

\section{EXPERIMENTAL RESULTS}

\section{A. Measurement of neutral sheet profiles}

It was found previously that driven reconnection in MRX generates an axisymmetric neutral sheet. ${ }^{6}$ It is natural to question whether the Harris equilibrium, which was derived as a 1-D equilibrium between a confining magnetic field and plasma pressure, could be observed and whether the magnetic reconnection process would affect the neutral sheet profiles given in Eqs. (12)-(17).

Precise magnetic field profiles of the MRX neutral sheet have been measured by a highresolution 1-D magnetic probe array, which contains magnetic pickup coils every $5 \mathrm{~mm}$ and is inserted radially across the neutral sheet. Figure 2 shows the time evolution of measured $B_{Z}(R)$ data, which are fitted to

$$
B_{Z}(R)=-B_{0} \tanh \left[\left(R-R_{0}\right) / \delta\right]+b_{1} R+b_{2},
$$

from which the current density $j_{\theta}$ is derived,

$$
j_{\theta}(R)=\frac{B_{0}}{\mu_{0} \delta} \operatorname{sech}^{2}\left[\left(R-R_{0}\right) / \delta\right] .
$$

The factors $b_{1}$ and $b_{2}$ are determined by the background quadrupole and equilibrium fields. The latter is applied to keep the plasma in a desirable position. The factor $b_{1}$ does not appear in Eq. (20) because it is cancelled exactly by $\partial B_{R} / \partial Z$ of the background quadrupole field. Note that the MRX coordinates $R, \theta$, and $Z$ correspond to $x, y$, and $z$ in the Harris model. Between $t \approx 260 \mu \mathrm{s}$ and $t \approx 275 \mu \mathrm{s}$, the reconnection layer is maintained in quasi steady-state. It should be noted that, as reported previously, ${ }^{5} B_{Z}$ data also fit well to an arctangent, leading to a Lorentzian profile for $j_{\theta}$. In our systematic studies, error functions (which lead to Gaussian $j_{\theta}$ ) were not as good as either hyperbolic tangents or arctangents. Since this paper focuses on the physical picture of the Harris model, only hyperbolic tangent 
fitting is shown. The difference between using Eqs. (19) and (18) for fitting is negligible due to the large aspect ratio $R_{0} \gg \delta$ in MRX.

The neutral sheet thickness $\delta$ can be determined accurately from the fit in Eqs. (19) and (20). By varying the discharge voltage and using both hydrogen and deuterium fill gas (to change the density and mass, respectively), a range of $\delta$ 's and $c / \omega_{\mathrm{pi}}$ 's were measured experimentally. Fig. 3(a) shows that the data match well with $\delta \approx 0.35 c / \omega_{\text {pi }}$, i.e. $\delta$ scales with $c / \omega_{\mathrm{pi}}$. The modified Harris theory gives a prediction for $\delta_{\text {Harris }}$, as given in Eq. (17). Experimentally, $\delta, c / \omega_{\text {pi }}$, and $\sqrt{2} V_{s} / V_{\text {drift }}$ are all determined independently, so we can compare $\delta$ versus $\delta_{\text {Harris }}$ Fig. $3(\mathrm{~b})$ shows good agreement between $\delta$ and $\delta_{\text {Harris }}$.

The 1-D Harris solution should satisfy MHD force balance, $j_{y} B_{z}=\nabla p$, which simplifies to $\left[p+B_{z}^{2} / 2 \mu_{0}=\right.$ constant $]$ if field line tension is small compared to magnetic pressure. Radial profiles of $n_{e}$ and pressure $p=n\left(T_{e}+T_{i}\right)$ in MRX have been measured to check this static force balance. The radial profiles of $B_{Z}(R), j_{\theta}(R)$ and $p(R)$ are shown in Fig. 4 . The third row of Fig. 4 plots the radial profile of $p+B_{Z}^{2} / 2 \mu_{0}$, which shows that MHD force balance is satisfied during the central time of reconnection but not at the beginning or at the end of the reconnection phase. In the early phase of reconnection $(t \lesssim 255 \mu \mathrm{s})$, magnetic pressure dominates the total pressure profile and reconnection drives low-beta plasma into the neutral sheet region. When plasma pressure increases to balance the magnetic pressure $(t \approx 255-272 \mu \mathrm{s})$, equilibrium is established to maintain quasi steady-state reconnection. In the Sweet-Parker model, this situation is described as a steady flow of plasma frozen into the magnetic field from the $R$ direction to the $Z$ direction. In MRX, strong ion heating ${ }^{16}$ and compressibility $^{7}$ create a state of excess kinetic pressure in the neutral sheet $(t \gtrsim 272 \mu \mathrm{s})$, breaking the pressure balance.

The excellent fit of MRX magnetic data points to the Harris profile and the agreement between measured and predicted $\delta$ are remarkable since the MRX plasma is undergoing reconnection. However, based on the discussions in Sec. II regarding the effects of dissipation, the excellent agreement indicates strongly that the Harris solution is the most natural one for a quasi steady-state reconnecting plasma sheet. Phenomenologically, since dissipation is 
related to an effective resistivity through Ohm's law along the current sheet, $\eta j_{y}=E_{y}-v_{x} B_{z}$, one expects that equilibrium in the $x$ direction $(R$ in MRX) can be maintained during the reconnection process provided $V_{x} \ll V_{\mathrm{A}}$, which is satisfied in $\operatorname{MRX}\left(V_{x} / V_{\mathrm{A}} \lesssim 0.1\right)$.

The precise determination of the magnetic field profile in MRX has enabled a detailed study of the neutral sheet thickness $\delta$ as a function of other relevant parameters. It is found that $\delta \sim c / \omega_{\mathrm{pi}}$. In anti-parallel reconnection cases on MRX, the ion gyro-radius $\rho_{i}$ is on the order of $c / \omega_{\mathrm{pi}}$. This indicates the importance of pressure balance (between $p$ and $B_{0}^{2} / 2 \mu_{0}$ ) and ion gyro-motion in determining the structure of reconnection region. This result is in rough agreement with numerical simulations ${ }^{10^{-13}}$ and observations in the geotail and the magnetopause. ${ }^{3}$ The detailed features of the neutral sheet profile provide a good indicator for the nature of magnetic reconnection.

\section{B. Enhanced ion heating and ion flows in the reconnection layer}

One of the most important local physics issues for magnetic reconnection is how magnetic energy is converted into plasma thermal energy. Figure 5 shows the time evolution of global $T_{i}$ and local $T_{e}$ during magnetic reconnection in helium discharges. These $T_{i}$ measurements are based on chord-averaged Doppler spectroscopy of the HeII $4686 \AA$ line. Plasma light is collected along a sightline located in the plane $Z=0 \mathrm{~cm}$ and tangent to the reconnection layer at $R=37.5 \mathrm{~cm}$. It is seen that the average $T_{i}$ rises initially together with $T_{e}$ but later surpasses $T_{e}$ by as much as a factor of three. It is difficult to obtain precise spectral measurements of $T_{i}$ in hydrogen discharges, although the hydrogen data show very similar trends. Recently, local ion heating due to reconnection has been identified ${ }^{16}$ on MRX using more rigorous measurements from a novel spectroscopy probe ${ }^{17}$ placed inside the neutral sheet. These results are reported in detail elsewhere. ${ }^{16}$ The significant ion heating was seen to be much larger than the values predicted by classical dissipation.

Mach probe measurements show downstream ion flow along the neutral layer $V_{i Z}(Z)$ (for $R=37.5 \mathrm{~cm})$, as shown in Fig. 6. Using an unmagnetized fluid sheath theory ${ }^{18}$ generalized 
for $T_{i} \gtrsim T_{e}$, it is shown that the maximum $V_{i Z} \lesssim 0.2 V_{A} \approx 10 \mathrm{~km} / \mathrm{s}$. The Mach probe measurements were calibrated using Doppler shifts obtained by the spectroscopy probe. The sub-Alfvénic flow is understood to result from high downstream pressure and moderate $\nabla p$ in the $Z$ direction. ${ }^{7}$ The flow speed is seen to increase linearly along the layer from $Z=0 \rightarrow 10 \mathrm{~cm}$. The peak flow velocity differs from classical MHD reconnection models, which predict Alfvénic ion outflows.

The observed enhanced ion heating cannot be due to thermalization of energetic flows. Instead, it is likely that the heating is caused by fluctuations which are also believed to play a dominant role in determining the plasma resistivity and ultimately the reconnection rate.

\section{SUMMARY AND DISCUSSION}

The neutral sheet profile during magnetic reconnection has been measured precisely in MRX. The magnetic field profile is seen to agree remarkably well with the Harris sheet profile even though the Harris theory does not take into account reconnection and associated dissipation. The measured neutral sheet thickness $\delta$ is seen to scale with the ion skin depth $c / \omega_{\mathrm{pi}}$ according to $\delta \approx 0.4 c / \omega_{\mathrm{pi}}$ over a wide range of discharge conditions. These results imply that that one can include the effects of quasi-steady state reconnection within the basic formulation of the Harris sheet. Because the Harris shifted Maxwellian velocity distribution function satisfies the full Fokker-Planck equation (leaving out the small electron-ion collision term), it appears also to be the natural state for a reconnecting plasma.

It is also found that a static force balance is maintained between incoming magnetic field and the neutral sheet plasma pressure during the quasi-steady state phase of reconnection. Deviation from pressure balance occurs in the beginning and ending phases without changing the magnetic profile shape from that of the Harris solution. It was found ${ }^{16}$ that a significant amount of magnetic energy is converted to thermal energy of the neutral sheet plasma during reconnection and that the energy conversion rate is much larger than the value expected from classical dissipation mechanisms. In earlier studies, it was found that a generalized Sweet- 
Parker model, which incorporates compressibility, downstream pressure and the effective resistivity, can explain the reconnection rate in MRX.,8

A significant implication of the MRX results to date is that a stable 2-D reconnection neutral sheet with axisymmetric geometry is consistent with both the Sweet-Parker and the Harris models with generalizations. Resistivity enhancement is observed during reconnection

in the collisionless regime. The relationship $\delta \approx 0.4 c / \omega_{\mathrm{pi}} \sim \rho_{\mathrm{i}}$ suggests that $V_{\mathrm{drift}}$ is limited to a value equal to a constant on the order of 3 or 4 times the ion thermal speed. ${ }^{8}$ These results lead us to suspect that a current-driven instability may be excited to limit the current. Immediate future efforts on MRX will be devoted to identifying the cause of the enhanced resistivity, including macro- and micro-instabilities.

\section{ACKNOWLEDGMENTS}

The authors acknowledge Mr. D. Cylinder for excellent technical support. This work was supported jointly by the National Aeronautics and Space Administration, the National Science Foundation, and the Department of Energy. 


\section{REFERENCES}

${ }^{1}$ V. M. Vasyliunas, Rev. Geophys. Space Phys. 13, 303 (1975).

${ }^{2}$ see e.g. E. N. Parker, in Cosmical Magnetic Fields (Clarendon Press, Oxford, 1979).

${ }^{3}$ see e.g. M. G. Kivelson and C. T. Russell, Introduction to Space Physics (Cambridge University Press, Cambridge, 1995).

${ }^{4}$ J. B. Taylor, Rev. Mod. Phys. 58, 741 (1986).

${ }^{5}$ M. Yamada, H. Ji, S. Hsu, T. Carter, R. Kulsrud, N. Bretz, F. Jobes, Y. Ono, and F. Perkins, Phys. Plasmas 4, 1936 (1997).

${ }^{6}$ M. Yamada, H. Ji, S. Hsu, T. Carter, R. Kulsrud, Y. Ono, and F. Perkins, Phys. Rev. Lett. 78, 3117 (1997).

${ }^{7}$ H. Ji, M. Yamada, S. Hsu, and R. Kulsrud, Phys. Rev. Lett. 80, 3256 (1998).

${ }^{8}$ H. Ji, M. Yamada, S. Hsu, R. Kulsrud, T. Carter, and S. Zaharia, Phys. Plasmas 6, 1743 (1999).

${ }^{9}$ E. G. Harris, Il Nuovo Cimento 23, 115 (1962).

${ }^{10}$ D. Biskamp, Phys. Fluids 29, 1520 (1986).

${ }^{11}$ R. Horiuchi and T. Sato, Phys. Plasmas 1, 3587 (1994).

${ }^{12}$ D. M. Uzdensky, Ph.D. Thesis, Princeton University (1998).

${ }^{13}$ M. A. Shay, J. F. Drake, R. E. Denton, and D. Biskamp, J. Geophys. Res. 103, 9165 (1998).

${ }^{14}$ S. M. Mahajan, Phys. Fluids B 1, 43 (1989).

${ }^{15}$ S. M. Mahajan and R. D. Hazeltine, submitted to Phys. Plasmas (1999).

${ }^{16}$ S. C. Hsu, G. Fiksel, T. A. Carter, H. Ji, R. M. Kulsrud, and M. Yamada, to be submitted 
(1999).

${ }^{17}$ G. Fiksel, D. J. D. Hartog, and P. W. Fontana, Rev. Sci. Instr. 69, 2024 (1998).

${ }^{18}$ M. Hudis and L. M. Lidsky, J. App. Phys. 41, 5011 (1970). 


\section{FIGURES}
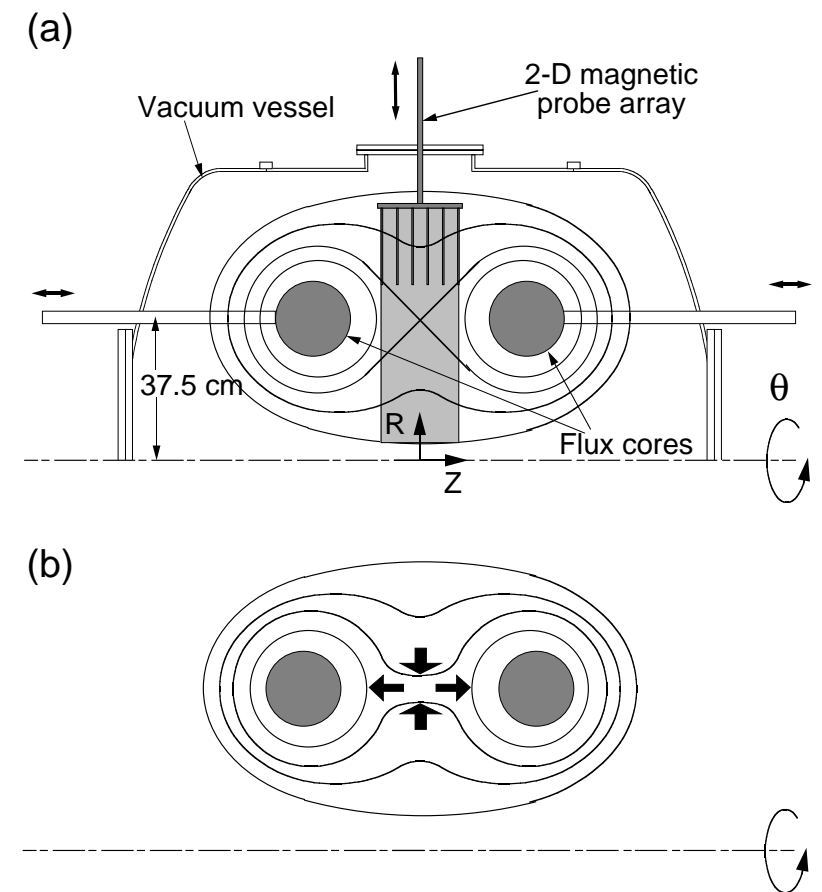

(c)

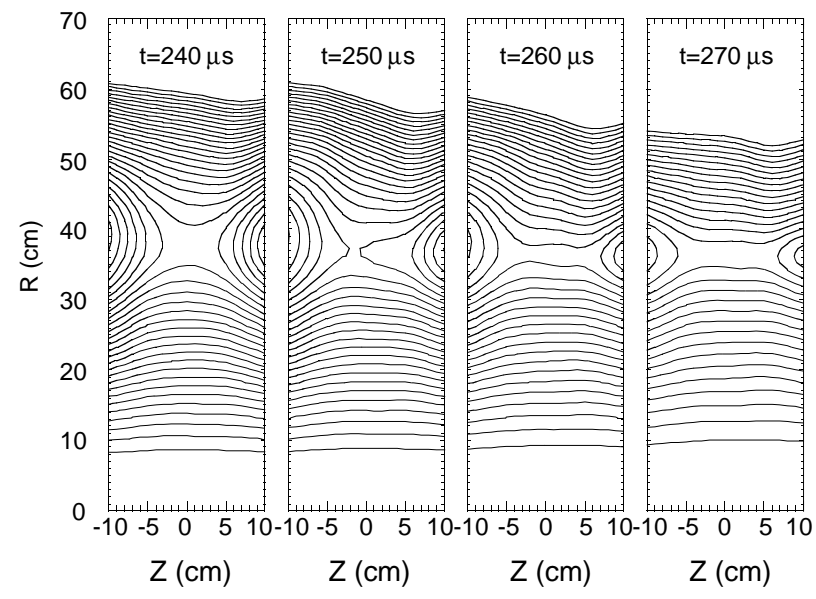

FIG. 1. (a) Experimental setup showing flux-cores and magnetic probe array. (b) Schematic of pull reconnection. (c) Time evolution of poloidal flux calculated from magnetic probe data, showing the development of a quasi steady-state neutral sheet. 


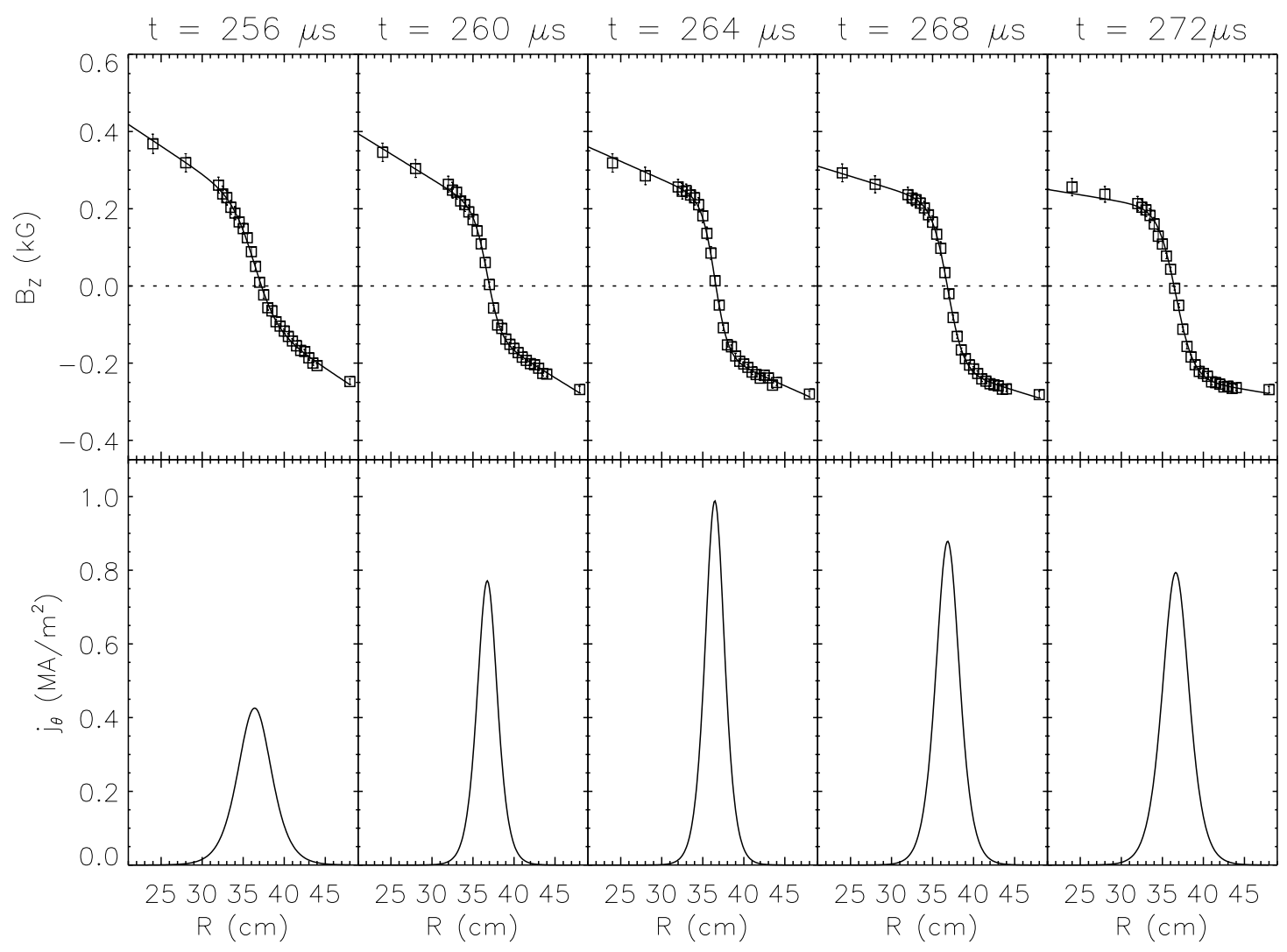

FIG. 2. Shown are reconnecting field (top) and current density (bottom) radial profiles taken in one plasma discharge. $B_{Z}(R)$ data are fit to the Harris sheet magnetic field profile modified by a background quadrupole field, Eq. (19), from which $j_{\theta}(R)$ is deduced, Eq. (20). 
(a)
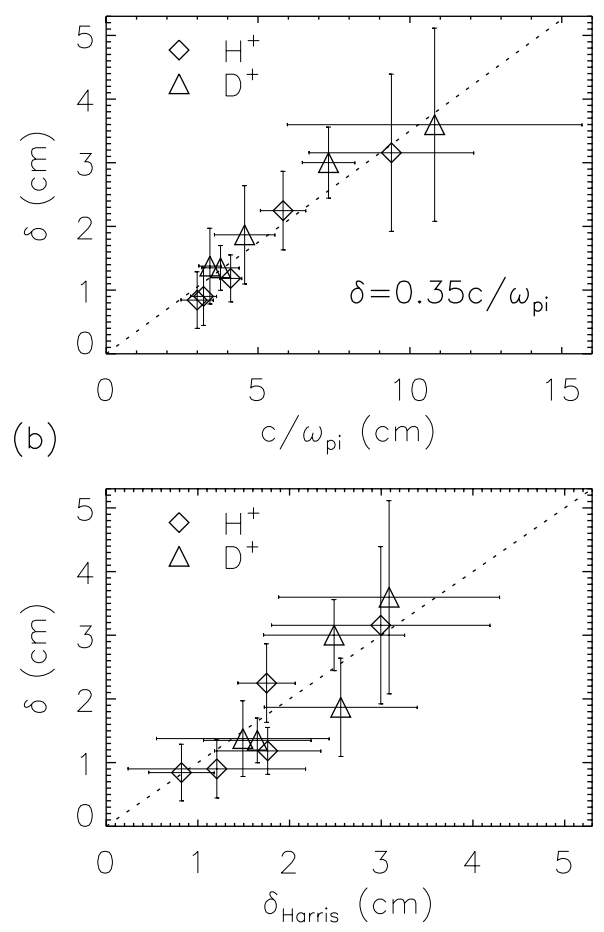

FIG. 3. Measured neutral sheet thickness $\delta$ versus (a) ion skin depth $c / \omega_{\text {pi }}$ and (b) versus theoretical Harris value given by Eq. (17). 


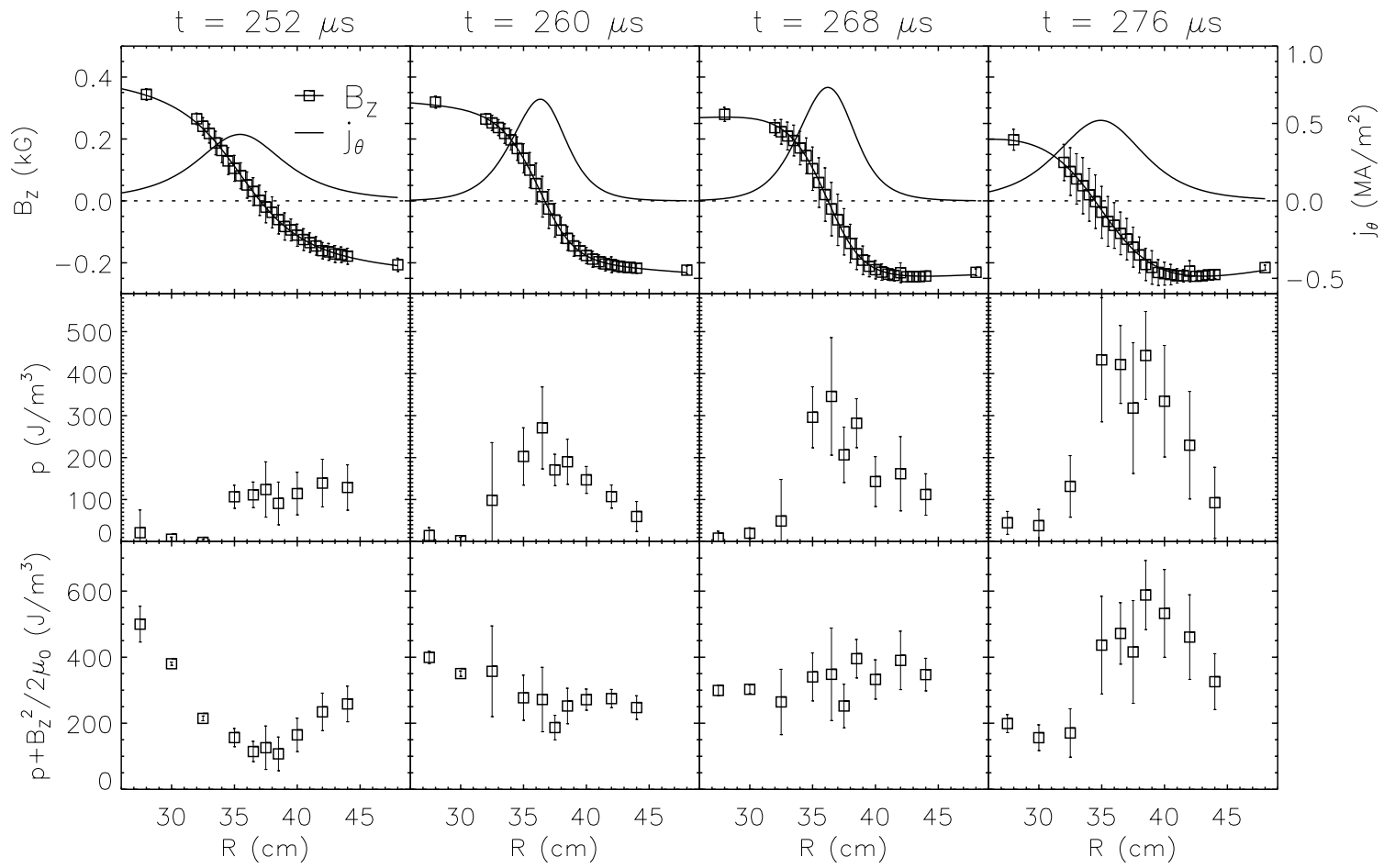

FIG. 4. Radial profiles of (top) reconnecting field, (middle) toroidal current density, and (bottom) plasma thermal plus magnetic field pressure. Static equilibrium is maintained from $t \approx 260-270 \mu \mathrm{s}$.

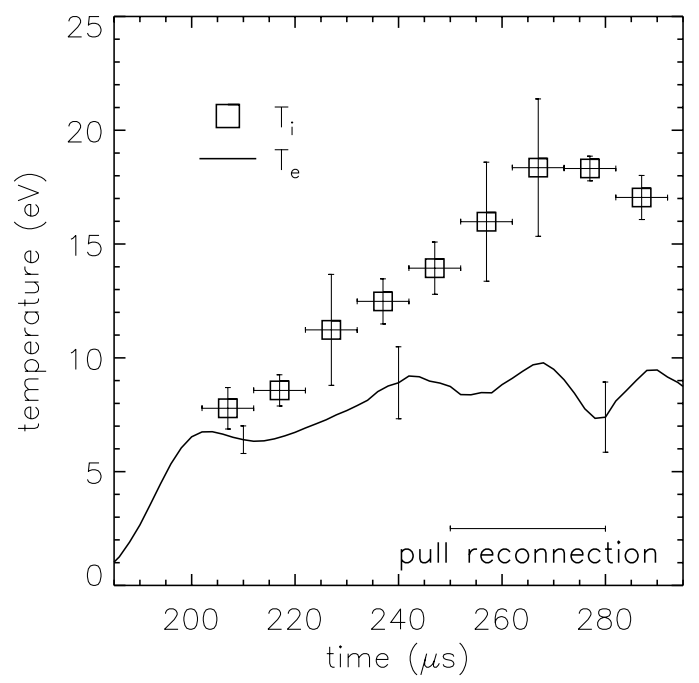

FIG. 5. Time evolution of global $T_{i}$ (measured using Doppler spectroscopy in helium discharges) and $T_{e}$, showing fast ion heating. 


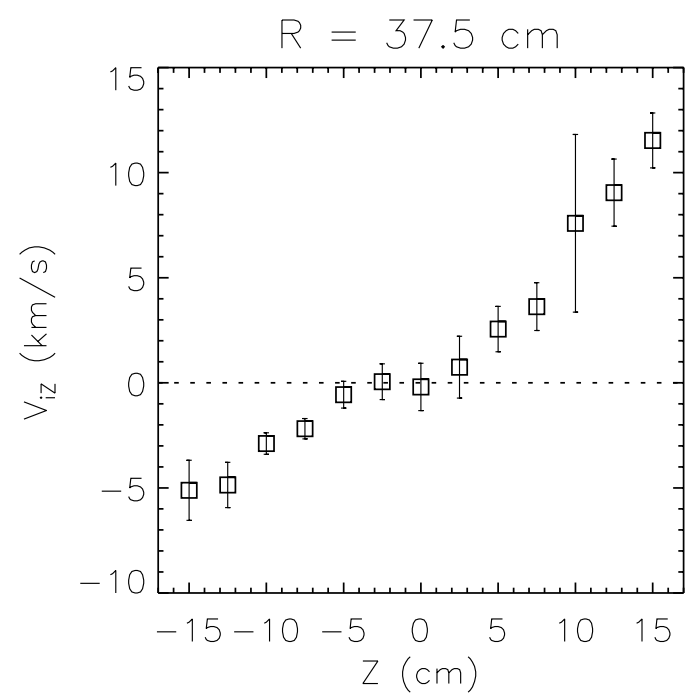

FIG. 6. Ion downstream flow speed along the neutral layer, as measured by a Mach probe, is established early during the reconnection phase, $t=250 \mu \mathrm{s}$. The flow remains sub-Alfvénic throughout the quasi steady-state pull reconnection phase $\left(V_{\mathrm{A}} \approx 60 \mathrm{~km} / \mathrm{s}\right)$. 